\title{
Towards Evidence-Based Management of Inherited Breast and Breast-Ovarian Cancer
}

\author{
Pål Møller \\ Section of Genetic Counseling, Department of Cancer Genetics, The Norwegian Radium Hospital, Oslo, Norway
}

Key words: inherited, breast cancer, ovarian cancer, BRCA, prevention

Corresponding author: Pål Møller, Section of Genetic Counseling, Department of Cancer Genetics

The Norwegian Radium Hospital, N-0310 0slo, Norway, e-mail:pal.moller@klinmed.uio.no

Submitted: 28 November 2003

Accepted: 10 December 2003

\begin{abstract}
Inherited breast-ovarian cancer was described in 1866. The underlying genetic defects in BRCA1/2 were demonstrated 128 years later. We now have 10 years of experience with genetic testing in BRCA kindreds. The majority of breast cancer kindreds (familial breast cancer) do not demonstrate ovarian cancer and are not associated with BRCA mutations. The effect of early diagnosis and treatment is monitored through international collaborations.

BRCA 1 -associated breast cancer is biologically different from other breast cancers, including a worse prognosis. BRCA2-associated breast cancer is, beside early onset, in many ways similar to sporadic breast cancer. Mammography screening of the high risk groups aiming at early diagnosis and treatment, seems promising for familial breast cancer and for BRCA2-associated breast cancer, but numbers included for BRCA2 carriers are limited. BRCA 1 -carriers have worse prognosis, and the potential benefit of MRI for early diagnosis is now being explored. Early diagnosis and treatment of ovarian cancer does not substantially improve survival, and prophylactic oophorectomy at the end of childbearing ages is advocated. Prophylactic mastectomy is debated, and we may await the results of MRI trials before recommending this option. Familial breast cancer and BRCA2-associated breast cancers are often oestrogen receptor positive, and may be prevented by oestrogen blockers/inhibitors. Oophorectomy prevents ovarian cancer, and may possibly prevent both receptor positive and receptor negative breast cancer as well, also while using HRT. Oral contraceptives may reduce ovarian cancer risk and increase breast cancer risk, irrespective of initial risk and genetic subgroup.
\end{abstract}

Inherited breast cancers are (pleiotropic) expressions of mutations in a number of distinct genes causing other cancers as well. To some extent, the biological function of the different genes is known, and to some extent the ways breast cancers are produced when this function is lacking, have been described. The different genetic causes lead to biologically different diseases. The cancers associated with different genetic syndromes occur at different ages, they may differ with respect to tumour characteristics, they have different prognosis, and they respond differently to prophylactic and treatment modalities. Following the clinical genetic work-up to provide health care to a woman possibly at risk for inherited breast cancer, there is a multistep approach. If she is demonstrated to be at risk, the preventive/treatment modalities she needs are a consequence of which subgroup of the inherited breast cancers she is at risk with. 
In most European countries, it is the understanding to discuss health care as what is to be offered to any woman who needs it. Thus, the question is not what might be done or what money possibly can buy - it is about what is reasonable and affordable. It is also about tradition and culture - what is possible in the current socio-ethical context. This contribution relates to these bearings.

The first detailed scientific description of inherited breast-ovarian cancer was given by Paul Broca in 1866 [1]. He demonstrated the transmission of the assumed underlying genetic defect, its expressions, the age-related and sex-limited penetrance, and the possibilities of modifying environmental and genetic factors. Not until 10 years ago, we learned that the syndrome is produced by BRCA1 mutations. BRCA2 mutations produce breast cancers in a similar prevalence and at a similar age, but with completely different tumour characteristics and with a different set of associated cancers. A number of additional genes cause multiorgan cancer syndromes when mutated, including mutations in TP53, PTEN, ATM and CHEK2. Germline mutations in TP53 and PTEN invoke childhood cancers. Heterozygous state for ATM mutation as a predisposition to breast cancer is debated, and the CHEK2 syndrome needs further evaluation. These rare genetic syndromes are not further discussed in this paper.

Some [2] assume that there have to be more dominantly inherited breast cancer genes, others disagree and conclude that they may be recessive, low penetrant or multifactorially interacting, but not dominantly inherited with high penetrance [3]. All cancer genetic clinics are aware that "inherited breast cancer" outside demonstrated BRCA mutation carrying syndromes may be a fiction, nevertheless all such clinics have defined a large volume of "inherited breast cancer" cases defined by family history but lacking demonstrated DNA mutations and referred them to follow-up examinations. We have demonstrated a BRCA mutation in but a small fraction of our at-risk families, and we have excluded BRCA mutations in a number of large dominantly inherited breast cancer pedigrees. There are genes not yet found.

None of the models for probability calculations for recurrence risk of breast cancer in breast cancer kindreds are valid after a BRCA mutation have been excluded they all assume that the patient is selected from an untested population. However, many cancer genetic centres continue to estimate the probability that a given woman is a mutation carrier, after she has been tested and found not to be so. This makes sense if you - as we do - believe there are more genes. It does not make sense if you do not believe so. Whatever we may believe, the calculations are wrong, because they cannot be interpreted without correcting the probability estimates for the testing performed prior to the calculations. Moreover, our activity of nesting up all the large mutation-carrying families will remove a substantial part of the mutation carriers from the population before the remaining familial clusters are referred to genetic counselling: for each passing day, the next familial cluster of breast cancer referred is less likely to harbour one of our founder BRCA1 mutations.

Several reports have estimated penetrance of BRCA mutations with respect to breast cancer. The results differ widely. This may in principle be due to four factors: (1) selection biases, (2) improper algorithms to calculate penetrances, (3) true differences between the mutations examined and/or (4) environmental or genetic modifiers of penetrance. Because the first series obviously had selection biases and because many of the families were not tested (carrier status for relatives was assumed, calculated upon and thereafter presented as results), the methodological problems may have been major. Studies based upon testing rather than assuming carrier status, and studies employing sophisticated statistics to eliminate methodological problems, now agree that penetrance for breast cancer is high for all BRCA 1 or BRCA2 truncating mutations [4-5].

Breast and ovarian cancers are competitive causes of death in a BRCA1/2 mutation carrier, and both are caused by the same mutation. Estimating the penetrance of breast cancer (or ovarian cancer) implies the methodological problem of informed censoring: whatever you do is methodologically wrong, because you censor the data with an argument dependent on what you are examining. Because there is no universally "correct" method, the solution is to formulate explicit questions specifying the assumptions to the answers. If a figure for probability for a healthy mutation carrier to contract ovarian cancer is looked for, one must censor out all mutation carriers when they contract another (breast) cancer. Doing so, lifetime penetrance for ovarian cancer is high in BRCA1 mutation carriers, possibly as high as for breast cancer. Two of the reasons for the low penetrance for ovarian cancer in some studies may be the combined effect that the families were selected for by the presence of breast cancer, and the fact that you may not contract ovarian cancer after having died of breast cancer. In addition, there may be biological differences between different mutations in the 
same genes. The exercise of pooling a number of small families without excluding the index cases used to ascertain the families, with different mutations, without actually testing the relatives but assuming their carrier status, without specifying the questions addressed and how the data are censored to answer that question, may give results of low practical value.

A statistical flaw has made some mistakenly conclude that BRCAl-associated cancers have prognosis similar to other breast cancers. The flaw is that according to oncological standards for randomized trials to evaluate effects of treatment modalities, the BRCA1 cancers have been compared to controls selected for similar prognostic tumour characteristics (oestrogen receptor, histopathological grade, etc.). In this way, BRCA1 cancers have been matched with a subset of patients demonstrated to have bad prognostic signs (over-parameterization). The question of whether BRCA1 mutation carriers have even worse prognosis is debated, but all studies agree that BRCA1 cancers have worse prognosis than age-matched controls [6-7]. Because a germline mutation is always prior to the tumour it causes, it is not meaningful to have the opposite as an alternative. However, most microarray studies present hierarchical trees with germline mutations as late events. Possibly, the mathematical models are pertinent, but the way the results are presented may seduce the readers to conceptional misunderstandings.

BRCA 1 -associated breast cancers are, as a group, different from all other defined groups of breast cancers [6-8]. The picture is so clear that all exceptions may be sporadic cancers caused by different mechanisms in BRCA1 mutations carriers (age-related sporadic breast cancer may occur in BRCA1 mutation carriers as well). The BRCA 1 -associated breast cancers are hormone receptor negative, of histopathological high grade, and they are close to never appear as precancers (DCIS) when diagnosed clinically or by mammography. Attempts of early diagnosis to achieve early treatment was initially considered successful: the tumours diagnosed were small and often without spread. It turned out, however, that the prognosis was not as good as hoped for according to the stage at diagnosis: retrospective series before any attempt on early diagnosis and treatment demonstrated 5 -year survival of $63 \%$ for invasive BRCA1 cancers [9]. The results of the Biomed2 prospective series included a 5 -year survival of $63 \%$ for invasive BRCA1 cancers, point estimate was no effect at all [7]. The conclusion was that annual clinical mammography for early detection did not work. Prophylactic mastectomy is an alternative [10-1 1], but with severe implications both on personal and professional ethical levels, and it is resource demanding. A new attempt on secondary prophylaxis has been implemented in most centres: MRI obviously has the capability of demonstrating tumours invisible in mammography [12]. We do not, however, at present know whether or not MRI may diagnose the tumours before they have biologically achieved their bad prognostic propensities. We hope it will work. All our BRCA1 mutation carriers are now subjected to annual MRI. Shortly we will have the early figure for their 5-year survival. We ask for time-out to retrieve this figure before we consider the alternatives.

Early diagnosis and treatment to improve prognosis for inherited ovarian cancer was undertaken by ultrasound and CEA125 in many centres. It did not work - no report claims substantial improvement in survival [13]. Actually, there are few reports on survival, most reports mention cancer with spread at diagnosis but give no survival data. However, it became clear that BRCA 1 -associated ovarian cancer seldom occurs before the age of 40 . Combined with the finding that oophorectomy at that age reduced not only ovarian cancer risk by more than $90 \%$, but also reduced breast cancer risk in BRCA1 mutation carriers - even in those using hormone replacement therapy [14] - most centres advocate prophylactic oophorectomy past childbearing ages [15]. In contrast to prophylactic mastectomy where uptake is low, the majority of postmenopausal BRCA1 carriers seem to choose oophorectomy. If a mean to early diagnosis and cure for ovarian cancer appears, we may soon have no BRCA 1 carriers left to evaluate the effects, because there may not be many ovaries left in the mutation carriers aged over 40 years. Moreover, the disease is so lethal and prophylactic oophorectomy past childbearing ages seems to be so well tolerated, that it would be hard to suggest a trial. In contrast to the differences in breast cancer phenotype, ovarian cancer caused by BRCA2 seems similar to that caused by BRCA 1, besides that the penetrance may be lower and disease onset later in BRCA2 carriers.

One report concluded that oophorectomy in BRCA1 carriers contracting cancer improved the prognosis of the breast cancer [7]. This observation needs to be supported by an independent series, but it is in keeping with the beneficial effect of prophylactic oophorectomy to breast cancer risk in the same group.

Breast cancers in inherited/familial non-BRCA1/2 carriers have good prognosis (about 90\% 5-year survival) inside early detection programmes applying 
Table 1. Primary and secondary prevention for women at risk for inherited breast or breast-ovarian cancer

BRCA1 mutation carriers (by testing or assumed by family history)

1. Annual clinical mammography and MRI from the age of 30 years onwards. For demonstrated mutation carriers there should be no upper age limit. Healthy at-risk women based on family history alone will have low probability for being mutation carriers over 70 years of age.

2. Prophylactic mastectomy should be an option, but may not be actively advocated until the benefit of MRI is known.

3. Prophylactic oophorectomy over 35 years of age when family is completed.

BRCA2 mutation carriers (by testing or assumed by family history)

1. As for BRCA1 mutation carriers, but oophorectomy may not be indicated before 40 years of age.

2. There is no consensus on practical use, but anti-oestrogens may prevent oestrogen receptor-positive tumours.

Familial breast cancer (high breast cancer risk according to family history not including ovarian cancer)

1. Annual clinical mammography from the age of 30 years onwards.

2. There is no consensus on practical use, but anti-oestrogens may prevent oestrogen receptor-positive tumours.

annual clinical mammography [7]. Moreover, a number of cases are demonstrated as precancers (DCIS), and they have reportedly 100\% event-free 5 year survival. Few studies have been presented on prospective survival in BRCA2-associated breast cancers, but these cancers seem to be comparable to sporadic breast cancers beside the young age of onset [8]. There is no indication that the prognosis should be worse than that of non-BRCA1/2-associated breast cancers.

Because all non-BRCAl carriers (including the BRCA2 carriers) predominantly contract hormone receptor-positive breast cancers, they should theoretically benefit from receptor blocking agents (like tamoxifen) and oestrogen production blocking agents (aromatase inhibitors). All available evidence indicate so. Most agree that by now it would be reasonable to suggest such chemoprevention to these groups, but there is no agreement on which compound and exactly which group to address. It is advocated to give various regimens of such treatment under strict control to evaluate the effects. That is where we are today: trials and discussions, but no consensus on applying chemoprevention as standard health care.

Again, the BRCA1-associated cancers differ. It may be expected that because as a group they have receptor-negative cancers, they should not respond either to oestrogen blockers or to oestrogen. Neither is true. In BRCA1 carriers, oophorectomy prevents breast cancer [14], tamoxifen prevents contralateral breast cancer [16], and oral contraceptives induce breast cancer [17]. Why and how is unclear. It seems, however, irrational to suggest oestrogen blockers to prevent oestrogen receptor-negative tumours.

In oncology, the scientific standard is a randomized trial. As is evident from the reports (beside chemoprevention) mentioned above, we have no randomized trials. You cannot randomize a woman to mastectomy, and you cannot deprive a mutation-carrying woman from any potential life-saving health care available. We are faced with the challenge of doing science without randomized trials, and we cannot (as the mammographic screening of older women) go and get the families we want for research. This leaves us with series subjected to a number of ascertainment biases, and we should interpret the results with caution. Because we are outside the framework of randomized trials, we may be better off not discussing exact figures in single reports, but rather focus on the main results, methods employed and whether or not the empirical facts are in keeping with the current paradigms for understanding. In this perspective, it may seem that we need to reconsider our paradigms for BRCA 1 -associated breast cancer. For the other groups, early diagnosis and treatment works as expected, improving early diagnosis may hopefully further improve the results obtained so far, and the principles of chemoprevention may be projected from sporadic cancers.

In conclusion to the facts discussed above, most clinical genetic centres relating to inherited breast cancer today advocate annual mammography from the age of 30 onwards to women at risk for inherited non-BRCA1 breast cancer. It is agreed that besides BRCA 1 carriers, oestrogen blockers/aromatase inhibitors may be beneficial - but there is no agreement on exactly how to implement such chemoprevention.

In contrast, annual mammography does not work in BRCAl carriers. We are now hoping MRI to be better to avoid large numbers of prophylactic mastectomies. We are confused by the data on the effect of hormones and hormone blockers in BRCA 1 carriers, and we all hope for chemoprevention to make the unpleasant discussion of prophylactic surgery superfluous if early detection and treatment does not work. Early diagnosis does not work for ovarian cancers, but oophorectomy is beneficial and advocated at the end of childbearing ages. 
As is the case with most scientific reports, this contribution has focused on unsolved problems. We may remember, however, that we actually have about $90 \% 5$-year survival of non-BRCA1 breast cancer with today's means [7], and the great majority of inherited breast cancers belong to this group. Prophylactic oophorectomy at the end of childbearing ages in BRCA1 carriers reduces morbidity and mortality by more than 50\% [14]. The systematic attempts to prevent and cure inherited breast cancer have been undertaken for but about 10 years. The results are actually good, which is reflected in the high compliance from the affected kindreds [18]. The attempts to prevent and cure inherited breast cancer is an example of a consumer-driven activity based on knowledge and collaboration from the patients needing our care. Actually, based on knowledge two BRCA1 carriers with small invasive tumours without spread this year have asked me for immediate chemotherapy. Educated patients may soon be our scientific collaborative partners. Trials we cannot impose upon them, may soon be initiated by patients who want to know.

The physicians and the researchers have the role of producing, filing, retrieving and communicating knowledge as appropriate. The choice what to do, however, should be the patient's choice, there are no scientific arguments as to whether or not to undergo prophylactic oophorectomy. As we all know, there are more arguments about what to do with your life than the doctors' suggestions. The high compliance to our advice during the last decade most probably reflects that the families have identified their problems long ago and were waiting for our care. The high compliance to genetic testing obviously reflects the opinion that our activity may prevent and cure. To maintain the high compliance, we should not over-sell expected results of interventions. We do not need to gain trust, we already have it. But we may lose it. Our role is not to make decisions. It is our obligation, however, to ensure that the advocated options are actually available to each single patient. In addition, we may produce arguments to advocate some options because they have consequences to our liking. The suggestions in Table 1 may be agreed by most, and some would go further and actively advocate prophylactic mastectomy in BRCA1 carriers and chemoprevention for the rest. Genetic counselling is to present information and options so that any given patient may be supported in exploring her values to make her choices. The challenge is to support the patients who make choices not corresponding with your own priorities.

\section{References}

1. Broca P. Traite des tumeurs. Paris 1866; 150-155.

2. Møller P, Borg A, Heimdal K, Apold J, Vallon-Christersson J, Hovig E, Maehle L, Nonwegian Inherited Ovarian Cancer Group. The BRCAl syndrome and other inherited breast or breastovarian cancers in a Norwegian prospective series. Europ J Cancer 2001; 37: 1027-1032.

3. Antoniou A, Pharoah PD, McMullan G, Day NE, Stratton MR, Peto J, Ponder BJ and Easton DF. A comprehensive model for familial breast cancer incorporating BRCA1, BRCA2 and other genes. Europ J Cancer 2002; 38: 76-83.

4. Antoniou A, Pharoah PD, Narod S, Risch HA, Eyfjord JE, Hopper $\mathrm{JL}$, Loman N, Olsson H, Johannsson O, Borg A, Pasini B, Radice P, Manoukian S, Eccles DM, Tang N, Olah E, Anton-Culver H, Warner E, Lubinski J, Gronwald J, Gorski B, Tulinius H, Thorlacius S, Eerola H, Nevanlinna $H$, Syrjakoski K, Kallioniemi OP, Thompson D, Evans C, Peto J, Lalloo F, Evans DG and Easton DF. Average risks of breast and ovarian cancer associated with BRCA1 or BRCA2 mutations detected in case series unselected for family history: a combined analysis of 22 studies. Am J Hum Genet 2003; 72: $1117-1130$.

5. Heimdal K, Mahle L, Apold J, Pedersen JC and Møller P. The Norwegian founder mutations in BRCA 1: high penetrance confirmed in an incident cancer series and differences observed in the risk of ovarian cancer. Europ J Cancer 2003; 39: 2205-2213.

6. Foulkes WD, Chappuis PO, Wong N, Brunet JS, Vesprini D, Rozen F, Yuan ZQ, Pollak MN, Kuperstein G, Narod SA and Begin LR. Primary node negative breast cancer in BRCA1 mutation carriers has a poor outcome. Ann Oncol 2000; 11 : 307-313.

7. Møller P, Borg A, Evans DG, Haites N, Reis MM, Vasen H, Anderson E, Steel CM, Apold J, Goudie D, Howell A, Lalloo F, Maehle L, Gregory H and Heimdal K. Survival in prospectively ascertained familial breast cancer: analysis of a series stratified by tumour characteristics, BRCA mutations and oophorectomy. Int J Cancer 2002; 101: 555-559.

8. Lakhani SR, van de Vijver MJ, Jacquemier J, Anderson TJ, Osin PP, McGuffog L and Easton DF. The pathology of familial breast cancer: predictive value of immunhistochemical markers, estrogen receptor, progesterone receptor, HER-2, and p53 in patients with mutations in BRCA1 and BRCA2. J Clin Oncol 2002; 20: 1310-1318.

9. Verhoog LC, Brekelmans CT, Seynaeve C, van den Bosch LM, Dahmen G, van Geel AN, Tilanus-Linthorst MM, Bartels CC, Wagner A, van den Ouweland A, Devilee P, Meijers-Heijboer EJ and Klijn JG. Survival and tumour characteristics of breast-cancer patients with germline mutations of BRCA 1. Lancet 1998; 351 : 316-321.

10. Meijers-Heijboer EJ, Verhoog LC, Brekelmans CT, Seynaeve C, Tilanus-Linthorst MM, Wagner A, Dukel L, Devilee P, van den Ouweland AM, van Geel AN and Klijn JG. Preventive strategies for hereditary breast or ovarian carcinoma differ from one country to another: differences among English, French and Canadian women. Cancer 2000; 92: 959-968.

11. Julian-Reynier CM, Bouchard LJ, Evans DG, Eisinger FA, Foulkes WD, Kerr B, Blancquaert IR, Moatti JP and Sobol HH. Women's attitudes toward presymtomatic DNA testing and prophylactic surgery in families with a BRCA1 or BRCA2 mutation. Lancet 2001; 355: 2015-2020.

12. Kuhl CK, Schmutzler RK, Leutner CC, Kempe A, Wardelmann E, Hocke A, Maringa M, Pfeifer U, Krebs D and Schild HH. Breast MR imaging screening in 192 women proven or suspected to be carriers of a breast cancer susceptibility gene: preliminary results. Radiology 2000; 215: 267-279. 
13. Maehle L, Apold J, Heimdal K and Møller P. Survival in prospectively detected inherited ovarian cancer. In: The Breast Cancer Linkage Consortium (BCLC) and the International Collaborative Group on Familial Breast and Ovarian Cancer (ICG- FBOC) 14 $4^{\text {th }}$ general meeting. Madrid, June 2-4, 2003. Benites J, Devilee P, Goldgar D, Eccles D, ed. Book of abstracts 2003; p 70.

14. Rebbeck TR, Lynch HT, Neuhausen SL, Narod SA, van't Veer L, Garber JE, Evans G, Isaacs C, Daly MB, Matloff E, Olopade OI, Weber BL; Prevention and Observation of Surgical End Points Study Group. Prophylactic oophorectomy in carriers of BRCA1 or BRCA2 mutations. N Engl J Med 2002; 346: 1616-1622.

15. Møller P, Evans G, Haites N, Vasen H, Reis MM, Anderson E, Apold J, Hodgson S, Eccles D, Olsson H, Stoppa-Lyonnet D, Chang-Claude J, Morrison PJ, Bevilacqua G, Heimdal K, Maehle L, Lalloo F, Gregory H, Preece P, Borg A, Nevin NC, Caligo M and Steel CM. Guidelines for follow-up of women at high risk for inherited breast cancer: consensus statement from the Biomed 2 Demonstration Programme on Inherited Breast Cancer. Dis Markers 1999; 15: 207-211.

16. Narod SA, Brunet JS, Ghadirian P, Robson M, Heimdal K, Neuhausen SL, Stoppa-Lyonnet D, Lerman C, Pasini B, de los Rios P, Weber B, Lynch H; Hereditary Breast Cancer Clinical Study Group. Tamoxifen and risk of contralateral hereditary breast cancer clinical study group. Lancet 2000; 356: 1876-1881.

17. Narod SA, Dube MP, Kliin J, Lubinski J, Lynch HT, Ghadirian $P$, Provencher D, Heimdal K, Moller P, Robson M, Offit K, Isaacs C, Weber B, Friedman E, Gershoni-Baruch R, Rennert G, Pasini B, Wagner T, Daly M, Garber JE, Neuhausen SL, Ainsworth P, Olsson H, Evans G, Osborne M, Couch F, Foulkes WD, Warner E, Kim-Sing C, Olopade O, Tung N, Saal HM, Weitzel J, Merajver S, Gauthier-Villars M, Jernstrom H, Sun P and Brunet JS. Oral contraceptives and the risk of breast cancer in BRCAl and BRCA2 mutation carriers. J Natl Cancer Inst 2002; 94: 1773-1779.

18. Bodd TL, Reichelt J, Heimdal K and Møller P. Uptake of BRCA1 genetic testing in adult sisters and daughters of known mutation carriers in Norway. J Genet Counselling 2003; 12: 405-417. 